Article

\title{
Correcting the Input Data for Calculating the Asymmetry of Hydrogenic Spectral Lines in Plasmas
}

\author{
Paul Sanders * and Eugene Oks *
}

Physics Department, 206 Allison Lab, Auburn University, Auburn, AL 36849, USA

* Correspondence: phs0007@auburn.edu (P.S.); goks@physics.auburn.edu (E.O.)

Received: 17 February 2018; Accepted: 2 March 2018; Published: 6 March 2018

\begin{abstract}
We provide corrections to the data in Sholin's tables from his paper in Optics and Spectroscopy 26 (1969) 27. Since his data was used numerous times by various authors to calculate the asymmetry of hydrogenic spectral lines in plasmas, our corrections should motivate revisions of the previous calculations of the asymmetry and its comparison with the experimental asymmetry, and thus should have a practical importance.
\end{abstract}

Keywords: asymmetry of spectral lines; corrections to input data; hydrogenic spectral lines in plasmas

The input data presented in Sholin's tables from paper [1] was used numerous times by various authors to calculate the asymmetry of hydrogenic spectral lines in plasmas. (For the latest advances in the theory of the asymmetry we refer to papers [2,3] and references therein). However, we found that there are incorrect entries tabulated in paper [1] for the the Ly- $\gamma, \mathrm{Ly}-\epsilon$, and $\mathrm{H}-\alpha$ lines, in both the intensity corrections and the quadrupole frequency corrections.

The dipole and quadrupole frequency corrections are given in paper [1] as

$$
\Delta_{k}^{\text {dipole }}=n q-n^{\prime} q^{\prime}
$$

and

$$
\Delta_{k}^{q u a d r u p}=\frac{1}{3}\left[n^{4}-n^{2}-6 n^{2} q^{2}-n^{\prime 4}+n^{\prime 2}+6 n^{\prime 2} q^{\prime 2}\right],
$$

where $n$ and $n^{\prime}$ are the principal quantum numbers of the upper and lower energy levels, respectively; $q=n_{1}-n_{2}$ and $q^{\prime}=n_{1}{ }^{\prime}-n_{2}{ }^{\prime}$ are the combinations of the corresponding parabolic quantum numbers.

\section{Frequency Corrections}

For Ly-gamma $(n=4)$, Equation (2) becomes:

$$
\Delta_{k}^{\text {quadrup }}(q)=80-32 q^{2}
$$

It yields $\Delta_{k}^{\text {quadrup }}(0)=80, \Delta_{k}^{\text {quadrup }}( \pm 1)=48, \Delta_{k}^{\text {quadrup }}( \pm 2)=-48, \Delta_{k}^{\text {quadrup }}( \pm 3)=-208$. The comparison shows that in Sholin's table there are typographic errors in $\Delta_{k}^{\text {quadrup }}(0)$ entered as 60 (instead of 80) and in $\Delta_{k}^{\text {quadrup }}( \pm 3)$ entered as -206 (instead of -208).

For Ly-epsilon $(n=4)$, Equation (2) becomes:

$$
\Delta_{k}^{q u a d r u p}(q)=420-72 q^{2}
$$

It yields $\Delta_{k}^{\text {quadrup }}(0)=420, \Delta_{k}^{\text {quadrup }}( \pm 1)=348, \Delta_{k}^{\text {quadrup }}( \pm 2)=132, \Delta_{k}^{\text {quadrup }}( \pm 3)=-228$, $\Delta_{k}^{\text {quadrup }}( \pm 4)=-732, \Delta_{k}^{\text {quadrup }}( \pm 3)=-1380$. The comparison shows that in Sholin's table there are typographic errors in $\Delta_{k}^{\text {quadrup }}( \pm 2)$ entered as 108 (instead of 132). 


\section{Intensity Corrections}

The intensity corrections are calculated from the corresponding corrections to the wave functions. The latter are given, e.g., in the Appendix of paper [4].

For H-alpha ( $n=3$ to $n=2$ transition), the comparison shows that in Sholin's table there are typographic errors in $\epsilon_{k}^{(1)}$ corresponding to $\Delta_{k}^{\text {dipole }}=2$ entered as -62 (instead of $\left.-62 / 9\right)$ and $\Delta_{k}^{\text {dipole }}=-2$ entered as 62 (instead of 62/9), as shown in detail below.

For $\Delta_{k}^{\text {dipole }}=2$ :

$$
\begin{aligned}
I_{k}=<110^{\prime \prime}|z| & 010^{\prime \prime} \\
& =\left(<110|z| 010>-3 \frac{a_{0}}{R}<200|z| 010>+3 \frac{a_{0}}{R}<020|z| 010\right. \\
& >-\frac{a_{0}}{R}<110|z| 100>+3 \frac{a_{0}{ }^{2}}{R^{2}}<200|z| 100>-3 \frac{a_{0}{ }^{2}}{R^{2}}<020|z| 100 \\
& >)^{2} \approx<110|z| 010>{ }^{2}-6 \frac{a_{0}}{R}<200|z| 010>+6 \frac{a_{0}}{R}<020|z| 010 \\
& >-2 \frac{a_{0}}{R}<110|z| 100>=I_{k}{ }^{(0)}\left(1-\frac{a_{0}}{R} \frac{62}{9}\right) .
\end{aligned}
$$

$$
\begin{aligned}
& \text { For } \Delta_{k}^{\text {dipole }}=-2 \text { : } \\
& \qquad \begin{aligned}
I_{k}=<110^{\prime \prime}|z| & 100^{\prime \prime}>^{2} \\
& =\left(<110|z| 100>-3 \frac{a_{0}}{R}<200|z| 100>+3 \frac{a_{0}}{R}<020|z| 100\right. \\
& >+\frac{a_{0}}{R}<110|z| 010>-3 \frac{a_{o}{ }^{2}}{R^{2}}<200|z| 010>+3 \frac{a_{o}{ }^{2}}{R^{2}}<020|z| 010 \\
& >)^{2} \approx<110|z| 100>^{2}-6 \frac{a_{0}}{R}<200|z| 100>+6 \frac{a_{0}}{R}<020|z| 100 \\
& >+2 \frac{a_{0}}{R}<110|z| 010>=I_{k}{ }^{(0)}\left(1+\frac{a_{0}}{R} \frac{62}{9}\right) .
\end{aligned}
\end{aligned}
$$

We note in passing that the robust perturbation theory, as developed by Oks and Uzer [5], allows for analytically calculating corrections to the eigenfunctions due to the quadrupole interaction in a much simpler way than in Sholin paper [1]. Details are presented in Appendix.

For completeness, we list below also previously known (for a long time) corrections to the tabulated entries from paper [1] for the H-beta line.

For the Stark components corresponding to the radiative transitions between the parabolic states 210 and 010 or between 120 and 100, the unperturbed intensity should be 81, instead of 16 .

For the Stark component corresponding to the radiative transition between the parabolic states 210 and 001 , the intensity correction $\epsilon_{k}{ }^{(1)}$ should be -20 (instead of -16 ).

For the Stark component corresponding to the radiative transition between the parabolic states 120 and 001 , the intensity correction $\epsilon_{k}{ }^{(1)}$ should be 20 (instead of 16).

There are also two corrections (known for a long time) to the following typographic errors from paper [1].

In Table 2 from [1] for the H-alpha line, in the header of the last column, the scaling factor should be $10^{6}$ instead of $10^{5}$.

In Equation (21) from [1], in its 2nd term in the right hand side, the coefficient should be $(3 / 8)$ instead of $(3 / 16)$. We note that after this correction, Equation (21) from [1] coincides with the corresponding term (proportional to 1/R ${ }^{4}$ ) in Equation (4.59) from book [5] after setting in the latter $Z_{1}=1, Z_{2}=Z$. Equation (4.59) from book [5] was derived from the exact expression for the energy in elliptical coordinates for the two Coulomb center problem by expanding the latter in powers of $1 / \mathrm{R}$ up to (including) the term $\sim 1 / \mathrm{R}^{6}$. Therefore, Equation (4.59) from book [5] can be considered, in particular, as the benchmark for testing Equation (21) from [1]. Such a test also confirms that the 2nd term in the right hand side of Equation (21) from [1] correctly contains the first power of $Z$ (while there were incorrect suggestions that this term should contain $\mathrm{Z}^{2}$ ).

In summary, since Sholin's input data from paper [1] was used numerous times by various authors to calculate the asymmetry of hydrogenic spectral lines in plasmas, our corrections should motivate 
revisions of the previous calculations of the asymmetry and its comparison with the experimental asymmetry, and thus should have a practical importance.

Acknowledgments: The authors are grateful to A.V. Demura for providing a valuable information.

Author Contributions: Both authors contributed equally to this work.

Conflicts of Interest: The authors declare no conflicts of interest.

\section{Appendix A. Application of the Robust Perturbation Theory [5] for Calculating Quadrupole Corrections to the Wave Functions}

Here, we use the robust perturbation theory [6]. The gist of it is as follows. If for the perturbed quantum system there is an operator $A$ that commutes with the Hamiltonian $H$ and the parts of these operators $A_{0}$ and $H_{0}$, characterizing the unperturbed quantum system, also commute, then the perturbation theory can be constructed in terms of the perturbation $\left(A-A_{0}\right)$ to the operator $A_{0}$, rather than in terms of the perturbation $\left(H-H_{0}\right)$ to the operator $H_{0}$. For calculating corrections to the wave functions (which are common for both $A_{0}$ and $H_{0}$ ), the advantage is that the eigenvalues of the operator $A_{0}$ are typically nondegenerate (in distinction to the eigenvalues of the operator $H_{0}$ ). Therefore, for calculating the first order corrections to the wave functions, it is sufficient to use the first order of the nondegenerate perturbation theory with respect to the perturbation $\left(A-A_{0}\right)$ and it would not involve infinite summations. In distinction, for calculating the same corrections in terms of the perturbation $\left(H-H_{0}\right)$, one would have to proceed to the second order of the degenerate perturbation theory, involving infinite summations.

Below as the operator $A$ we choose the projection $A_{z}$ of the super-generalized Runge-Lenz vector, derived by Kryukov and Oks [7], on the axis connecting the nucleus of the hydrogenic atom/ion with the perturbing ion. The operator of the unperturbed projection $A_{z}{ }^{(0)}$ has the well-known eigenvalues $q / n$-see. e.g., the textbook [8]. According to Equation (12), from [6], the first non-vanishing term of the expansion of the operator $\left(A_{z}-A_{z}{ }^{(0)}\right)$ in terms of the small parameter $n^{2} / R$ (here and below we use atomic units) is $-L^{2} / R$. Then, the corrections to the wave functions are given by

$$
-\frac{1}{R} \frac{\left(L^{2}\right)_{n q m}^{n q^{\prime} m}}{A_{z, \alpha}(0)-A_{z, \alpha^{\prime}}(0)}=-\frac{n}{R} \frac{\left(L^{2}\right)_{n q m}^{n q^{\prime} m}}{\left(q-q^{\prime}\right)}
$$

where the selection rules for non-zero matrix elements of the operator $L^{2}$ require $q-q^{\prime}= \pm 2$.

The non-diagonal matrix elements of the operator $L^{2}$ in parabolic coordinates (as well as of the operators $L_{ \pm}=L_{x} \pm \mathrm{i} L_{y}$ ), have been calculated by Sholin, Demura, and Lisitsa in [9]:

$$
\begin{aligned}
& <n_{1}+1, n_{2}-1, m\left|L^{2}\right| n_{1} n_{2} m>=-\left[n_{2}\left(n-n_{2}\right)\left(n_{1}+1\right)\left(n-n_{1}-1\right)\right]^{1 / 2} \\
& <n_{1}-1, n_{2}+1, m\left|L^{2}\right| n_{1} n_{2} m>=-\left[n_{1}\left(n-n_{1}\right)\left(n_{2}+1\right)\left(n-n_{2}-1\right)\right]^{1 / 2}
\end{aligned}
$$

We note that matrix elements of the operator $L_{x}$ in parabolic coordinates have been later reproduced by Gavrilenko in paper [10]. We also note that the non-diagonal matrix elements of the operators $L_{ \pm}$can also be obtained using their proportionality (within the manifold of the fixed $n$ ) to the non-diagonal matrix elements of the operators $(x \pm i y)$ :

$$
<n, q \pm 2, m\left|L_{ \pm}\right| n q m>=-( \pm 1)[2 /(3 n)](x \pm i y)
$$


(The underlying physical reason for the existence of relation (A3) is, according to Demura [11], the $\mathrm{O} 4$ symmetry of hydrogenic atoms/ions. ${ }^{1}$ ) Therefore, the non-diagonal matrix elements of the operator $L^{2}$ in parabolic coordinates can be obtained using their similar proportionality to the non-diagonal matrix elements of the operator $\left(x^{2}+y^{2}\right)$. The latter matrix elements have been calculated by Clark [12].

Anyway, after substituting the non-diagonal matrix elements of the operator $L^{2}$ from Equation (A2) in Equation (A1), the latter equation yields the following result for the corrections to the wave functions (more rigorously, for the coefficients of the corresponding linear combinations of the unperturbed wave functions):

$$
\begin{gathered}
\frac{n\left[n_{2}\left(n-n_{2}\right)\left(n_{1}+1\right)\left(n-n_{1}-1\right)\right]^{1 / 2}}{2 R}, q-q^{\prime}=2, \\
-\frac{n\left[n_{1}\left(n-n_{1}\right)\left(n_{2}+1\right)\left(n-n_{2}-1\right)\right]^{1 / 2}}{2 R}, q^{\prime}-q=2 .
\end{gathered}
$$

This is the same result as in Sholin paper [1], but it is obtained in a simpler way: without the need to go to the second order of the perturbation theory.

\section{References}

1. Sholin, G.V. On the nature of the asymmetry of the spectral line profiles of hydrogen in a dense plasma. Opt. Spectrosc. 1969, 26, 275-282.

2. Djurovic, S.; Ćirišan, M.; Demura, A.V.; Demchenko, G.V.; Nikolić, D.; Gigosos, M.A.; González, M.A. Measurements of $\mathrm{H}_{\beta}$ Stark central asymmetry and its analysis through standard theory and computer simulations. Phys. Rev. E 2009, 79, 046402. [CrossRef] [PubMed]

3. Demura, A.V.; Demchenko, G.V.; Nikolic, D. Multiparametric dependence of hydrogen Stark profiles asymmetry. Europ. Phys. J. D 2008, 46, 111-127. [CrossRef]

4. Bacon, M.E. The asymmetry of Ly- $\alpha$ and Ly- $\beta$. J. Quant. Spectrosc. Radiat. Transf. 1976, 17, 501-512. [CrossRef]

5. Komarov, I.V.; Ponomarev, L.I.; Slavjanov, S.Y. Spheroidal and Coulomb Spheroidal Functions; Nauka: Moscow, Russia, 1976. (In Russian)

6. Oks, E.; Uzer, T. A robust perturbation theory for degenerate states based on the exact constants of the motion. Europhys. Lett. 2000, 49, 554-557. [CrossRef]

7. Kryukov, N.; Oks, E. Supergeneralized Runge-Lenz vector in the problem of two Coulomb or Newton centers. Phys. Rev. A 2012, 85, 054503. [CrossRef]

8. Landau, L.D.; Lifshitz, E.M. Quantum Mechanics; Pergamon: Oxford, UK, 1965.

9. Sholin, G.V.; Demura, A.V.; Lisitsa, V.S. Electron impact broadening of Stark sublevels of a hydrogen atom in a plasma. 1972, Preprint IAE-2232. (In Russian)

10. Gavrilenko, V.P. Resonant modification of quasistatic profiles of spectral lines of hydrogen in a plasma under the influence of noncollinear harmonic electric fields. Sov. Phys. JETP 1991, 92, 624-630.

11. Demura, A.V. Private communication, 2018.

12. Clark, C.W. Case of broken symmetry in the quadratic Zeeman effect. Phys. Rev. A 1981, 24, 605. [CrossRef]

(C) 2018 by the authors. Licensee MDPI, Basel, Switzerland. This article is an open access article distributed under the terms and conditions of the Creative Commons Attribution (CC BY) license (http://creativecommons.org/licenses/by/4.0/).

1 Specifically, this is related to the following two facts within the manifold of the fixed $n$ [11]. First, the mean value $<\mathbf{r}>$ of the radius vector of the bound electron is proportional to the unperturbed Runge-Lenz vector $\mathbf{A}^{(0)}$, as it is well-known. Second, the linear combinations $\mathbf{J}_{ \pm}=\left(\mathbf{L} \pm \mathbf{A}^{(0)}\right) / 2$ obey the same commutation relations as the angular momentum. 